\title{
Ocupational therapy using Virtual Reality
}

\author{
José Rodrigues \\ LAETA - INEGI \\ University of Porto \\ Porto, Portugal \\ jose.c.rodrigues@gmail.com
}

\author{
Paulo Menezes \\ ISR \\ University of Coimbra \\ Coimbra, Portugal \\ paulo@isr.uc.pt
}

\author{
Maria Teresa Restivo \\ LAETA - INEGI \\ University of Porto \\ Porto, Portugal \\ trestivo@gcloud.fe.up.pt
}

\begin{abstract}
This work describes a system for occupational therapy based on a traditional pedal system instrumented with a simple sensor for providing the rotating speed value and Oculus Rift headset and their hand trackers all to be used interactively with a Virtual Reality application. A virtual city is used as the environment to be explored by the user. The present level of development is described as well as the types of stimulations it allows, some project decisions and how to implement the evaluation of user's performance.
\end{abstract}

Keywords—virtual reality, occupational therapy, serious games, active ageing, user experience

\section{INTRODUCTION}

Physical activity and cognitive stimulation have been identified as major practices in active and healthy aging processes. Continuous physical and mental stimulation can contribute to postpone several age related problems and can improve quality of life. Health professionals recommend performing simple daily tasks to elderly people, in order to stay active and prevent losses in muscular tissue and bone density. Tasks like walking can also help with cognitive stimulation when reading signs or names, listening sounds that can trigger memories, too $[1,2]$.

Unfortunately, at an advanced age people can stay inactive for long periods of time, which will decrease muscle mass significantly reducing mobility and independence. This can lead in long periods during a day of inactivity, for example sitting in front of a TV screen or sleeping. Health professionals such as occupational therapists recommend performing simple activities in order to improve fundamental skills. This type of therapies can be done in therapy centres, daily senior centres but can also be done independently at home if there is no significant risk. The development of such type of therapies are important, namely in the present moment the world is crossing - the pandemic period that is preventing people to contact freely with others, to go for short walks, etc., increasing all the negative aspects of loneliness [3].

The current work proposes using Virtual Reality (VR) based on traditional and low cost equipment for occupational therapy exercises that can be used either at home or in daily or therapy facility centres in order to increase motivation and treatment efficiency [4]. The reported VR therapy exercises can be performed with no direct constant supervision of a therapist, after the user familiarization. The application itself can integrate different kinds of therapy devices in the future and it allows evaluating the user performance. At the present, the application integrates different types of activities that can be used to provide the user with physical (for upper and lower limbs and head) and cognitive stimulation. It allows the user experiencing of a serious game type including different exercises either mental of physical. Data from the user performance can be stored in a cloud database for futures analysis or comparison of the user's evolution [5].

\section{OBJECTIVE}

This work presents and discusses an expandable VR framework consisting of a virtual city that supports the integration of different activities. Each of this activities are placed in a predetermined location within the virtual city, and offering one specific goal or activity, that can be either a physical or cognitive stimulation. This approach enlarges activity diversity reducing monotony and increasing motivation for the occupational therapy exercises. The user can freely explore the virtual world at his/her own speediness, choosing which task he wants to do, or following the therapist recommendations. This approach is similar to some retention strategies frequently explored in video games.

The tasks can gradually adapt to the user performance, and increase or decrease difficulty to create new challenges in order to consistently and gradually make the patient better at these activities and consequently better in his/her life. These tasks presented to the user are simple and can be easily recognized as a daily tasks, they are designed according to its specific therapeutic purposes, which can be cognitive stimulation or physical stimulation or both. As example, the user can be asked to go from a point $\mathrm{A}$ to a point $\mathrm{B}$, following directions or paths, according with road signs or road marks and a map of the virtual environment. Other tasks may include more physical activity characteristic, that can be simple arm movement, riding a bicycle or walking - for this example, the task should be quickly interpreted by the user and should have a quick real-time simulation in the virtual world through the integration of an avatar, making them easily recognisable and adaptable.

\section{MATERIALS AND METHODS}

\section{A. Software Tools and Development}

Based on a generic 3D city model, work was developed in order to optimize its complexity using Blender [6]. This software was also used to create the collision mesh to be applied to the model. Finally, these two meshes were integrated together in the Unity [7] game engine. The Unity software package facilitates the physics calculations, the integration of hardware, and the 3D virtual city app to create the present virtual reality application. The Unity libraries simplify the integration of the Oculus Rift headset in what concerns the visualization of virtual objects according to the user movements, and a pair of touch controllers and their associated constellation sensors to track hand movements.

To promote physical exercise, a sit-down traditional pedal system was associated with this VR app as shown in Figure 1. 
A rotating system, attached to the main axis of the pedals, with Bluetooth communication, was added and so it enables the communication of the activity-related data with the compter. The velocity at which the user is pedaling and its direction (forward or backward) are used by the app to animate user movement.

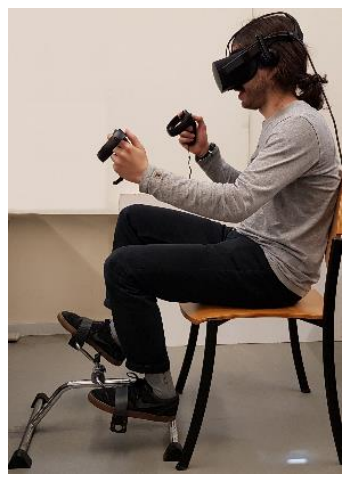

Fig. 1. System under use.

Some design decisions taken during the development of the user interaction were implemented to minimize dizziness and nausea while using the application - for example, to include a minimal amount of text or using some objects bigger than normal. Additionally, inspired by the natural people reaction while riding a bicycle, the users should tilt their heads while turning during the city exploration.

\section{B. The Application}

The application aims to create cognitive and visual stimulation, while the user is performing physical exercise. More than being taken on a tour along the city, the application enables the user to explore the city following his/her curiosity. To stimulate this curiosity and contribute to engage the user in an exercise that will enable traveling, and simultaneously provide cognitive stimulation there are different goals spread around the city to be reached,

The game interaction is done via a sit-down pedal, a lowcost traditional accessory for physical stimulation or rehabilitation equipment recommended by therapists for patients to have at home. This equipment is traditionally considered boring by the users. Using this device equipped with a Bluetooth sensor to determine the cadence and speed at which the user is pedaling, and connecting it to the application, we can transform the user motion in a simulated bicycle ride into the virtual environment.

While using the Oculus Rift and the touch controllers the user can observe and explore the surrounding environment, perform tasks and making some decisions. Since both the Head Mounted Display (HMD) and the touch controllers can be tracked using their associated constellation sensors it is possible to know the hands and head position and orientation of the user, allowing to create different types of activities and interactions with the application.

The user while riding the bicycle will need to use the sitdown pedal to move forward and then by tilting is head left or right, will turn the bicycle accordingly. This approach has to main effects: to provide neck muscles physical activity and to reduce the nausea and dizziness while using the application. For cognitive stimulation there are different signs spread around the city to inform the user which direction he should follow to reach his destination.

At the present, other tasks are available in the city like go shopping for fruit or going to the cinema. In the shopping task it is requested the user to buy a specific amount of different kinds of fruit. To be able to pick it up fruit for a basket the participant will need to use the touch controllers to track his/her hands position that will be represented in the virtual world. With this, the user interacts with the virtual world just like in the real world, where he needs to grab the fruit pieces with his/her hands, and place them in a fruit basket, forcing the user to perform physical activity in the arms and counting fruit pieces.

\section{RESULTS AND DISCUSSION}

A preliminary study of an early development of this VR app for Occupational Therapy has been already published [8].

According the data it was well accepted, and the participants felt motivated to use this application saying that they would use it, or a similar type of application, if available to them.

\section{CONCLUSIONS}

The results foster to carry on the present development and a few strategies will be implemented to improve problems in usability that were found in the preliminary study, as well new approaches to reduce side effects while using VR. Those have been the two main problems found.

\section{ACKNOWLEDGMENT}

Authors gratefully acknowledge the funding of Project NORTE-01-0145-FEDER-000022 - SciTech - Science and Technology for Competitive and Sustainable Industries, cofinanced by Programa Operacional Regional do Norte (NORTE2020), through Fundo Europeu de Desenvolvimento Regional (FEDER). This work was also funded by Project LAETA - UID/SEM/50022/2013, Fundação para a Ciência e Tecnologia.

\section{REFERENCES}

[1] D.I. Miller, V. Taler, P.S. Davidson and C. Messier, "Measuring the impact of exercise on cognitive aging: methodological issues." Neurobiology of aging, Vol .33, pp.622-e29, March 2012

[2] B. Hurley and I. Reuter, "Aging, physical activity, and disease prevention." Journal of aging research, , Vol. 2011, id 782546, April 2011.

[3] B. Gavin, J. Lyne and F. McNicholas." Mental Health and the COVID19 Pandemic". Ir. J. Psychol. Med., Vol. 74, pp.1-7, 2020 doi:10.1017/ipm.2020.72.

[4] R.V. Kenyon, J. Leigh and E. A. Keshner, "Considerations for the future development of virtual technology as a rehabilitation tool", Journal of NeuroEngineering and Rehabilitation, Vol.1, pp. 13, 2004 doi:10.1186/1743-0003-1-13

[5] F. Carneiro, R. Tavares, J. Rodrigues, P. Abreu and M.T. Restivo,"A gamified approach for hand rehabilitation device". Int. J. Online

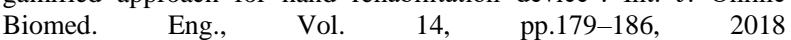
doi:10.3991/ijoe.v14i01.7793.

[6] https://www.blender.org/

[7] https://unity3d.com/

[8] J. Rodrigues, T. Coelho, P. Menezes and M. T. Restivo, "Immersive Environment for Occupational Therapy: Pilot Study", Information 2020, 11(9), 405; https://doi.org/10.3390/info11090405 